\title{
Proceeding
}

Supplementary Issue: Rio 2016 Olympic Games Second Anniversary Special Edition. Olympic Studies Forum, 2-3 October 2018.

Federal University of Espirito Santo, (Vitória - Espirito Santo), Brazil

\section{Olympic Values Inventory (IVO): Validation of an instrument for social sports programs}

\author{
ALESSANDRA SCARTON ${ }^{1,2}$, GABRIEL MERLIN ${ }^{1,4}$, TIAGO DA COSTA $^{1,2}$, NELSON TODT $^{1,2}$, \\ RICARDO SALDANHA ${ }^{1,3}$ \\ ${ }^{1}$ Olympic Studies Research Group, Pontifical Catholic University of Rio Grande do Sul (PUCRS), Porto \\ Alegre, Brazil \\ 2School of Health Sciences (Physical Education), Pontifical Catholic University of Rio Grande do Sul \\ (PUCRS), Porto Alegre, Brazil \\ ${ }^{3}$ La Salle University Centre, Canoas, Brazil \\ ${ }^{4}$ German Sports University Cologne, Cologne, Germany
}

\begin{abstract}
There is an increasing number of social sports programmes in Brazil which use values as an educational tool. In this sense, it is important to have an instrument capable of measuring these programmes efficiency. This paper aims to create and test the metrical principles of Content Validation Coefficient (CVC) of the Olympic Values Inventory through items that embrace the Olympic Values (Friendship, Respect and Excellence). The inventory items elaboration was based on three-dimensional structures, researchers' previous experiences and bibliographical review about the topic; which resulted in 41 items. To evaluate the CVC, five specialists have participated of the evaluation by analysing the clarity and relevance of each item based on a Likert scale (1 to 5). The results were assessed based on the Content Validation Coefficient calculation $\left(C V C_{t}\right)$. The $\mathrm{CVC}_{t}$ of the items was sufficient on both Clarity $\left(\mathrm{CVC}_{t}=0,79\right)$ and Relevance $\left(\mathrm{CVC}_{\mathrm{t}}=0,86\right)$. Therefore, it is considered that the Olympic Values Inventory is legitimate according to Content Validation. Keywords: Olympic values; Content validation; Psychometry; Sports values; Olympism.
\end{abstract}

\section{Cite this article as:}

Scarton, A., Merlin, G., Da Costa, T., Todt, N., \& Saldanha, R. (2019). Olympic Values Inventory (IVO): Validation of an instrument for social sports programs (in Portuguese). Journal of Human Sport and Exercise, 14(3proc), S371-S380. doi:https://doi.org/10.14198/ihse.2019.14.Proc3.10

Corresponding author. Avenida Ipiranga, 6681, CEP: 90619-900. Porto Alegre, Brazil.

E-mail: alescarton@pucrs.br

Supplementary Issue: Rio 2016 Olympic Games Second Anniversary Special Edition. Olympic Studies Forum, 2-3 October 2018. Federal University of Espírito Santo, (Vitória - Espírito Santo), Brazil.

JOURNAL OF HUMAN SPORT \& EXERCISE ISSN 1988-5202

(c) Faculty of Education. University of Alicante

doi:10.14198/jhse.2019.14.Proc3.10 


\section{INTRODUÇÃO}

Em 1894 Pierre de Coubertin propõe a primeira edição dos Jogos Olímpicos da Era Moderna, baseado nos quesitos da filosofia do Ginásio Grego visando à formação integral do homem através das práticas esportivas (Müller \& Todt, 2015). O presente estudo é uma parte de um projeto maior que visa construção de uma ferramenta capaz de verificar, identificar e analisar Valores Olímpicos (VOs) em iniciativas sócio esportivas.

Os VOs são concebidos pelas expressões 'Excelência, Amizade e Respeito' e possuem representações e significados dos ideais do Olimpismo. Deste modo, segundo estes valores possuem significado próprio, permitindo sua compreensão e associação com os símbolos Olímpicos: Excelência - Lema Olímpico; Amizade - Chama Olímpica; Respeito - Aros Olímpicos.

Cada Valor Olímpico, conforme mencionado, possui um importante significado, de acordo com a IOC (2007). O lema olímpico, Citius, Altius, Fortius (mais rápido, mais alto e mais forte), encontra associação com o valor 'Excelência', referente ao esforço e a busca pela melhor condição humana. Por sua vez, o sentido do valor 'Respeito', representado através dos Aros Olímpicos, faz referência ao comportamento íntegro e ético. Relaciona-se com este valor o conceito de Fair Play e o sentido de reciprocidade que permite assim a compreensão do adversário como ser essencial para que prática esportiva possa existir. E o terceiro valor, 'Amizade', representado pela Chama Olímpica sugere a concepção do esporte como forma de unir os povos, apesar das diferenças existentes, sejam elas econômicas, religiosas, culturais e raciais, ou de outra natureza.

Estes três Valores (tradicionais do Olimpismo), segundo Abreu (2002), foram interpretados como 'Valores Nucleares' proclamados pelo Comitê Olímpico Internacional (COI), considerando o sentido universal dos VOs. Através de uma rápida análise da atual sociedade brasileira, é possível identificar indícios suficientes para o reconhecimento de episódios de desestabilização na construção ou transmissão de valores e formação humana. Essa 'crise de valores' que a sociedade atual atravessa parece afastar sentimentos como fraternidade, cooperação, união e compartilhamento ao passo que se fortalece uma globalização que expõe os detalhes das desigualdades sociais evidenciadas com a consolidação de um sistema predominantemente opressor, pautado essencialmente, mas não exclusivamente, pelo vínculo econômico (Todt, 2015).

Nesse sentido, ancorar a formação integral de cidadãos comprometidos e interessados com o bem comum e dotados de valores éticos e morais que pautem a boa convivência em sociedade, passa a ser uma alternativa dentro da perspectiva da Educação Olímpica. Qual o impacto de usar o Olimpismo e seus valores de maneira pedagógica na formação de jovens? De que forma seria possível mensurar o aprendizado destes valores?

O que se percebe na atualidade, tendo como ponto de partida o período pré e pós Jogos Olímpicos Rio 2016, é o crescimento de projetos sociais esportivos no país que utilizam valores como meio de ensino. Em função da realidade social atual e dos desafios propostos pela Agenda Olímpica 2020, a tradição histórica do Olimpismo propõe-se a ser adotada e adaptada por algumas instituições do mundo moderno que incentivam atitudes éticas, ajustando o Movimento Olímpico ao nosso tempo. Para Todt (2015), a renovação de valores tem se mostrado crucial para disseminar uma educação baseada nos Valores Olímpicos.

Diante dessa compreensão acerca do Olimpismo e seu 'caminho' trilhado até os dias atuais, cabe melhor compreender os aspectos relacionados ao conceito de valores. Este conceito, conforme a literatura especializada (Perron, 1987; Rokeach, 1981; Schwartz, 2007; Schwartz \& Cieciuch, 2016), é concebido 
como modos de conduta ou estados de preferência. Nesse sentido, o 'valor', muitas vezes, torna-se necessário para determinada ação (atitude). Para Perron (1987), a definição operacional dos valores passa por três elementos constitutivos básicos: o cognitivo, o afetivo e o conativo.

Para 0 autor, os valores são abstrações oriundos de vivências acumuladas e tem uma função normativa para a avaliação de situações de envolvimento da pessoa com o ambiente físico e humano que a cerca. Do ponto de vista cognitivo, os valores representam estas abstrações aos quais são interpretados como modalidades de ser ou agir e finalidades de existência.

A desejabilidade está presente no elemento afetivo dos valores. É o nível de atração que a realidade exerce sobre a pessoa (bom ou ruim, verdadeiro ou falso, melhor ou pior) e, como consequência, o seu nível de investimento afetivo. Este atributo passa por uma análise fenomenológica do 'dever': (a) o que é socialmente exigido; (b) pessoalmente exigido e; (c) objetivamente apropriado. A percepção do 'dever' deriva da própria natureza da atividade ou de regras que fazem parte da sua essência.

O elemento conativo tem relação com os aspectos comportamentais. Trata-se de uma variável mediadora que conduz à ação, diante de uma multidão de opções de escolhas e tem coloração do desejável. No esporte não é difícil identificar situações desta natureza, como por exemplo, a de um jogador que perdeu uma partida ou um final de campeonato e decide cumprimentar seu adversário. Será que aquela ação sofre influência das regras sociais do jogo ou são crenças duradouras do próprio sujeito?

Diante do exposto, percebe-se que o construto 'valores' é uma dimensão conceitual extremamente complexa e pressupõe-se a existência de instrumentos válidos e fidedignos para medi-lo adequadamente (Saldanha, Balbinotti \& Balbinotti, 2015). A hierarquização de valores pelo grau de importância remete à possibilidade de mensuração e hierarquização dos sistemas de valores das pessoas (Rokeach, 1981, Saldanha, Balbinotti \& Balbinotti, 2015, Schwartz, 2007, Schwartz \& Cieciuch, 2016). Os valores são acontecimentos privados e, embora não sejam diretamente observáveis, podem ser identificados pelo comportamento verbal (ou descritivo) no momento da comunicação.

O Brasil recebe reconhecimento mundial como um país Olímpico em 2016, com os Jogos Olímpicos do Rio de Janeiro, porém, o caminho para sediar os Jogos começou a ser conduzido anos atrás. O Rio 2016 marca o fechamento da década do esporte no Brasil, depois de o país ser sede dos Jogos Pan-Americanos de 2007, dos Jogos Mundiais Militares de 2011, da Copa das Confederações de 2013 e da Copa do Mundo de 2014. Embora diversos projetos educativos e sociais envoltos pelo tema 'Olímpico' tenham proliferado no Brasil nesses últimos anos, observa-se a ausência de processos que avaliem seu desenvolvimento e resultados. Nesse sentido, faz-se necessário um instrumento confiável a fim de ser capaz de medir, psicometricamente, os Valores Olímpicos (Amizade, Respeito e Excelência).

Tendo em vista o crescimento "Olímpico" do Brasil nessa década e o aumento progressivo de projetos esportivos sociais e educacionais sobre o tema, o Grupo de Pesquisa em Estudos Olímpicos (GPEO) da Pontifícia Universidade Católica do Rio Grande do Sul começou em 2009 a elaboração de um questionário com o intuito de identificar os Valores Olímpicos, inicialmente intitulado Questionário de Valores Olímpicos (QVO-27) (Secco et al., 2009). A partir de 2012 o instrumento iniciou seu processo de construção e validação, passando a se chamar Inventário de Valores Olímpicos (Merlin et al., 2012), nome que perdura até hoje. Desse ano em diante 0 instrumento passou por outros processos de validação e aprimoramento de consistência interna até a versão atual (Scarton et al., 2016; Merlin et al., 2016a; Merlin et al., 2016b). 
Nesse sentido, o presente estudo tem por objetivo analisar os princípios métricos de Validade de Conteúdo do Inventário de Valores Olímpicos (IVO).

\section{PROCEDIMENTOS METODOLÓGICOS}

As etapas que compõem um processo de construção de instrumentos de medidas são de extrema importância. Nesse sentido, a primeira etapa consiste na elaboração da estrutura conceitual, na definição do construto, da sua dimensionalidade e do contexto na qual o instrumento está inserido sustentando 0 desenvolvimento dos domínios. Para a construção desse instrumento tomou-se como ponto de partida os Valores Olímpicos propostos pelo COI. Resumidos em: Amizade, Excelência e Respeito. Ao verificar documentos produzidos pelo Comitê Olímpico Internacional (IOC, 2007) foram encontradas as seguintes definições para os valores Amizade, Excelência e Respeito, respectivamente: compreensão de que 0 esporte pode ser um instrumento para o entendimento mútuo entre as pessoas de todo o mundo; entendida como a possibilidade de sempre fazer o melhor possível, levando em conta todos os aspectos da vida, valorizando a participação, mais do que a vitória; e compreendido como o respeito da própria da pessoa, do seu corpo, com as outras pessoas, com o esporte, regras e regulamentos e, além disso, com o meio ambiente.

A segunda e terceira etapas consistiram na definição do objetivo e da população envolvida. Nessa perspectiva 0 instrumento tem como proposta avaliar os Valores Olímpicos (Amizade, Excelência e Respeito) em participantes de projetos esportivos sociais, com idade igual ou superior a 12 anos.

Na quarta etapa ocorreu a construção e organização dos itens para posterior definição do tipo de escala de resposta e estruturação da mesma. Segundo Coluci, Alexandre e Milani (2015) os itens de uma escala não devem ser construídos ao acaso, e sim elaborados em função das definições operacionais do construto, que no caso do instrumento em questão, consiste nos Valores Olímpicos propostos pelo COI. Para a construção dos itens, foi definido o aspecto de clareza e pertinência.

Tendo conhecimento das etapas citadas acima, foi realizado o processo de construção do inventário. Em reunião, os pesquisadores responsáveis produziram uma lista de frases (itens), em torno do conceito central (debriefing), tendo por base a experiência dos investigadores e revisão de literatura sobre a temática. Procurando utilizar como critérios fundamentais a clareza de linguagem, a compreensão simples e a representatividade temática (pertinência), deste processo resultou uma lista de 41 itens diretamente relacionados aos Valores Olímpicos de Amizade, Respeito e Excelência.

Ainda nesse processo foi destacada a escolha do layout e do tipo de escala de resposta compreendidas enquanto quesitos de fundamental importância para a elaboração de um instrumento de medida (Hill \& Hill, 2008, Moraes, Hasselmann \& Reichenheim, 2002, Pasquali, 2001). Para os estudos de valores, as escalas devem apresentar opções de níveis de atribuição de importância e um número de pontos mais recomendado para 0 estudo. Frequentemente, em estudos de valores, utilizam-se escalas de 5 a 7 pontos (de "pouquíssimo importante" a "muitíssimo importante"). Além do tipo de escala, Schwartz (2007) recomenda que em estudos cross-culturais a escala para medir valores contenha pontos negativos, para que seja possível medir valores negativos (de "oposto aos meus valores" a "de suprema importância"). Para o IVO foi definida uma escala tipo likert de 5 pontos incluindo questões que medem valores positivos e negativos. 
Para os autores as primeiras impressões são importantes, então, recomenda-se que se faça uma pequena introdução no cabeçalho (apresentação do instrumento e instruções para o preenchimento) escrevendo-a de forma clara e que seja esteticamente atraente.

Após o cumprimento das etapas anteriores, a quinta, e última, etapa foi a de validação de conteúdo. A avaliação de conteúdo é essencial no processo de desenvolvimento de novos instrumentos de medidas porque representa o início de mecanismos para associar conceitos abstratos com indicadores observáveis e mensuráveis (Coluci, Alexandre, \& Milani, 2015). Para tanto, a amostra foi composta por 5 juízes avaliadores. O número de avaliadores seguiu as recomendações estabelecidas por Hernandez-Nieto (2002). Os critérios de escolha foram: (a) grau, mínimo, de Mestrado na área da Psicologia ou Educação Física; e (b) experiência de campo com a população alvo do questionário não inferior a 5 anos. Assim, dois dos avaliadores possuíam graduação em psicologia e com mestrado na área do esporte e, um terceiro, era graduado e com mestrado na área da Educação Física.

A fim de proceder a validação de conteúdo do IVO foram criadas, conforme modelo descrito na Tabela 1, duas escalas. Estas escalas, graduadas de 1 a 5, eram direcionadas para a avaliação da clareza e da pertinência de cada item. Além disso, era permitido aos avaliadores, um julgamento qualitativo, no espaço 'Observação', a respeito da atribuição da sua nota ao item, a fim de contribuírem para melhorá-lo.

Tabela 1. Instruções para o "juiz avaliador" responder ao questionário de aprovação do instrumento

\begin{tabular}{cccc}
\hline Itens & Clareza de linguagem & Pertinência & Observação \\
\hline Item 1 & $1|2| 3|4| 5$ & $1|2| 3|4| 5$ & \\
Item 2 & $1|2| 3|4| 5$ & $1|2| 3|4| 5$ & \\
Item 3 & $1|2| 3|4| 5$ & $1|2| 3|4| 5$ & \\
Item ... & $1|2| 3|4| 5$ & $1|2| 3|4| 5$ & \\
\hline
\end{tabular}
Nota. Adaptado de Cassepp-Borges, Balbinotti \& Teodoro (2010).

Assim, a partir destas notas em relação a clareza e pertinência, a próxima etapa foi calcular os índices de validade. Para tanto, o Quadro 1 apresenta a fórmula de Hernandez-Nieto (2002).

Quadro 1. Etapas da fórmula do Coeficiente de Validade de Conteúdo (CVC)

Descrição da Etapa

Segunda etapa - utilizando a média das notas é calculado o coeficiente de validação de conteúdo $\left(C V C_{i}\right)$, pega-se a média e divide pelo resultado máximo que a questão poderia receber, conforme escala de 1 a 5.

Terceira etapa - é preciso calcular possíveis erros sistemáticos, para ser descontado $\left(\mathrm{Pe}_{\mathrm{i}}\right)$. Para isso divide-se um pelo número de juízes elevado pelo mesmo número de juízes.

Quarta etapa - já pode ser calculado o $\mathrm{CVC}_{\text {final, }}$ utilizando o $\mathrm{CVC}_{\mathrm{i}}$ e subtraindo o erro sistemático. O CVC final representa o resultado de cada questão.

Quinta etapa - consiste em subtrair a média do CVCtotal de todas as questões pelas médias de erro sistemático. $0 \mathrm{CVC}_{\text {total }}$ representa o resultado final de toda o questionário.

\section{Cálculo Utilizado}

$$
\begin{gathered}
M_{x}=\frac{\sum_{i=1}^{J} x_{i}}{C V C_{i}}=\frac{M_{x}}{V_{\text {máx }}} \\
P e_{i}=\left(\frac{1}{J}\right)^{J} \\
C V C_{c}=C V C_{i}-P e_{i} \\
C V C_{t}=M c v c_{i}-M p e_{i}
\end{gathered}
$$

$$
\text { Nota. Adaptado de Hernandez-Nieto (2002). }
$$

\section{RESULTADOS}


A partir das respostas dos juízes avaliadores, procedeu-se a exploração dos resultados sobre o instrumento - IVO, segundo os cálculos de Hernandez-Nieto (2002) apresentados anteriormente. A partir das análises das respostas dos avaliadores, foi possível realizar o cálculo do CVC para cada item. A tabela abaixo apresenta os escores dos cálculos de clareza e pertinência de cada item, assim como o escore total $\left(C V C_{t}\right)$ das duas análises.

Com base na tabela acima, percebe-se que, em relação a clareza, $24,4 \%$ das questões apresentaram notas (CVC) abaixo de 0,70 . Cerca de $29,3 \%$ apresentaram resultados entre 0,70 e 0,80 . A maioria dos itens, $46,3 \%$, apresentaram resultados de $\mathrm{CVC}_{c}$ superiores a 0,80 . Em relação a pertinência, apenas $7,3 \%$ dos itens apresentaram índices de $\mathrm{CVC}_{\mathrm{c}}$ abaixo de 0,70 . Cerca de $22 \%$ apresentaram resultados entre $0,70 \mathrm{e}$ 0,80 de $\mathrm{CVC}_{\mathrm{c}}$. Entretanto, 70,7\% dos itens apresentaram índices de $\mathrm{CVC}_{\mathrm{c}}$ superiores a 0,80.

Conforme Tabela 2, nota-se que, de forma geral, a média das respostas dos avaliadores no critério Pertinência foi maior que a da Clareza. $O$ resultado do $\mathrm{CVC}_{\mathrm{t}}$ Pertinência foi de 0,86 , enquanto o de Clareza foi de 0,79 , considerado acima do ponto de corte estabelecido pela literatura. Cabe ressaltar que na avaliação da pertinência, apenas dois itens apresentaram valores de CVCp inferiores a 0,70 (itens 38 e 41) e dois itens apresentaram valores igual a 1,00 (itens 14 e 30). Este último resultado representa que todos os avaliadores, ao responderem a escala de pertinência, marcaram o máximo resultado, representado por "muitíssimo pertinente". Na avaliação da clareza, dez itens apresentaram valores inferiores a 0,7 (itens 6, 8, $10,13,21,24,25,31,32$ e 38) e nenhum item atingiu a nota máxima para a clareza. Diante desses resultados e dos comentários dos juízes, alguns itens foram modificados, conforme apresentados no Quadro 2.

Tabela 2. Coeficiente de Validade de Conteúdo (CVC)

\begin{tabular}{ccccc|ccccc}
\hline \multicolumn{5}{c|}{ CVC - Clareza } & \multicolumn{5}{c}{ CVC - Pertinência } \\
\hline Item & Média & $\mathrm{CVC}_{\mathrm{i}}$ & $\mathrm{Pe}_{\mathrm{i}}$ & $\mathrm{CVC}_{\mathrm{c}}$ & Item & Média & $\mathrm{CVC}_{\mathrm{i}}$ & $\mathrm{Pe}_{\mathrm{i}}$ & $\mathrm{CVC}_{\mathrm{c}}$ \\
\hline 1 & 3.6 & 0.72 & 0.00032 & 0.72 & 1 & 4.4 & 0.88 & 0.00032 & 0.88 \\
2 & 4.2 & 0.84 & 0.00032 & 0.84 & 2 & 4.0 & 0.80 & 0.00032 & 0.80 \\
3 & 4.2 & 0.84 & 0.00032 & 0.84 & 3 & 4.4 & 0.88 & 0.00032 & 0.88 \\
4 & 4.4 & 0.88 & 0.00032 & 0.88 & 4 & 4.8 & 0.96 & 0.00032 & 0.96 \\
5 & 3.6 & 0.72 & 0.00032 & 0.72 & 5 & 3.6 & 0.72 & 0.00032 & 0.72 \\
6 & 2.8 & 0.56 & 0.00032 & 0.56 & 6 & 4.2 & 0.84 & 0.00032 & 0.84 \\
7 & 4.0 & 0.80 & 0.00032 & 0.80 & 7 & 4.2 & 0.84 & 0.00032 & 0.84 \\
8 & 2.4 & 0.48 & 0.00032 & 0.48 & 8 & 4.2 & 0.84 & 0.00032 & 0.84 \\
9 & 4.2 & 0.84 & 0.00032 & 0.84 & 9 & 4.0 & 0.80 & 0.00032 & 0.80 \\
10 & 3.0 & 0.60 & 0.00032 & 0.60 & 10 & 3.4 & 0.68 & 0.00032 & 0.68 \\
11 & 4.4 & 0.88 & 0.00032 & 0.88 & 11 & 4.8 & 0.96 & 0.00032 & 0.96 \\
12 & 4.4 & 0.88 & 0.00032 & 0.88 & 12 & 4.4 & 0.88 & 0.00032 & 0.88 \\
13 & 3.4 & 0.68 & 0.00032 & 0.68 & 13 & 4.8 & 0.96 & 0.00032 & 0.96 \\
14 & 4.8 & 0.96 & 0.00032 & 0.96 & 14 & 5.0 & 1.00 & 0.00032 & 1.00 \\
15 & 4.0 & 0.80 & 0.00032 & 0.80 & 15 & 4.2 & 0.84 & 0.00032 & 0.84 \\
16 & 4.2 & 0.84 & 0.00032 & 0.84 & 16 & 4.2 & 0.84 & 0.00032 & 0.84 \\
17 & 3.8 & 0.76 & 0.00032 & 0.76 & 17 & 4.4 & 0.88 & 0.00032 & 0.88 \\
18 & 3.8 & 0.76 & 0.00032 & 0.76 & 18 & 4.2 & 0.84 & 0.00032 & 0.84 \\
19 & 4.0 & 0.80 & 0.00032 & 0.80 & 19 & 4.4 & 0.88 & 0.00032 & 0.88 \\
20 & 4.0 & 0.80 & 0.00032 & 0.80 & 20 & 4.4 & 0.88 & 0.00032 & 0.88 \\
\hline
\end{tabular}




\begin{tabular}{|c|c|c|c|c|c|c|c|c|c|}
\hline 21 & 3.2 & 0.64 & 0.00032 & 0.64 & 21 & 4.0 & 0.80 & 0.00032 & 0.80 \\
\hline 22 & 4.2 & 0.84 & 0.00032 & 0.84 & 22 & 4.0 & 0.80 & 0.00032 & 0.80 \\
\hline 23 & 4.0 & 0.80 & 0.00032 & 0.80 & 23 & 4.6 & 0.92 & 0.00032 & 0.92 \\
\hline 24 & 3.4 & 0.68 & 0.00032 & 0.68 & 24 & 4.6 & 0.92 & 0.00032 & 0.92 \\
\hline 25 & 3.0 & 0.60 & 0.00032 & 0.60 & 25 & 4.8 & 0.96 & 0.00032 & 0.96 \\
\hline 26 & 4.2 & 0.84 & 0.00032 & 0.84 & 26 & 4.4 & 0.88 & 0.00032 & 0.88 \\
\hline 27 & 4.8 & 0.96 & 0.00032 & 0.96 & 27 & 4.2 & 0.84 & 0.00032 & 0.84 \\
\hline 28 & 4.2 & 0.84 & 0.00032 & 0.84 & 28 & 4.8 & 0.96 & 0.00032 & 0.96 \\
\hline 29 & 4.2 & 0.84 & 0.00032 & 0.84 & 29 & 3.8 & 0.76 & 0.00032 & 0.76 \\
\hline 30 & 4.4 & 0.88 & 0.00032 & 0.88 & 30 & 5.0 & 1.00 & 0.00032 & 1.00 \\
\hline 31 & 3.4 & 0.68 & 0.00032 & 0.68 & 31 & 4.0 & 0.80 & 0.00032 & 0.80 \\
\hline 32 & 2.8 & 0.56 & 0.00032 & 0.56 & 32 & 3.8 & 0.76 & 0.00032 & 0.76 \\
\hline 33 & 4.0 & 0.80 & 0.00032 & 0.80 & 33 & 4.2 & 0.84 & 0.00032 & 0.84 \\
\hline 34 & 4.2 & 0.84 & 0.00032 & 0.84 & 34 & 4.2 & 0.84 & 0.00032 & 0.84 \\
\hline 35 & 4.8 & 0.96 & 0.00032 & 0.96 & 35 & 4.8 & 0.96 & 0.00032 & 0.96 \\
\hline 36 & 4.6 & 0.92 & 0.00032 & 0.92 & 36 & 4.8 & 0.96 & 0.00032 & 0.96 \\
\hline 37 & 4.2 & 0.84 & 0.00032 & 0.84 & 37 & 3.8 & 0.76 & 0.00032 & 0.76 \\
\hline 38 & 3.2 & 0.64 & 0.00032 & 0.64 & 38 & 3.4 & 0.68 & 0.00032 & 0.68 \\
\hline 39 & 3.8 & 0.76 & 0.00032 & 0.76 & 39 & 4.4 & 0.88 & 0.00032 & 0.88 \\
\hline 40 & 4.8 & 0.96 & 0.00032 & 0.96 & 40 & 4.8 & 0.96 & 0.00032 & 0.96 \\
\hline 41 & 3.8 & 0.76 & 0.00032 & 0.76 & 41 & 3.4 & 0.68 & 0.00032 & 0.68 \\
\hline & \multicolumn{3}{|c|}{ Total (CVC t Clareza) } & 0.79 & & \multicolumn{3}{|c|}{ Total (CVCt Pertinência) } & 0.86 \\
\hline
\end{tabular}

Quadro 2. Apresentação dos itens modificados

\begin{tabular}{|c|c|c|}
\hline Item & $1^{\text {a }}$ Versão & Itens Modificados \\
\hline 3 & Fazer amigos & Fazer novos amigos nas aulas/treinos \\
\hline 4 & Me esforçar para ganhar nos jogos & Me esforçar para ganhar \\
\hline 5 & Jogar bem nos treinos e campeonatos & Me superar nos treinos/aulas \\
\hline 6 & $\begin{array}{l}\text { Ter prioridade melhorar meu } \\
\text { desempenho nos jogos }\end{array}$ & $\begin{array}{l}\text { Ter como prioridade melhorar meu desempenho nos } \\
\text { campeonatos }\end{array}$ \\
\hline 13 & Demonstrar que sou o melhor nos jogos & Demonstrar que sou melhor que os outros \\
\hline 14 & Não desistir de jogos que estou perdendo & Não desistir quando estou perdendo \\
\hline 15 & Aprender novas habilidades & Aprender novos movimentos \\
\hline 20 & Não responder aos árbitros & Não desrespeitar os árbitros \\
\hline 21 & Não usar expressões inadequadas & $\begin{array}{l}\text { Não usar expressões inadequadas (palavrões e } \\
\text { xingamentos) }\end{array}$ \\
\hline 22 & Ajudar a manter o ambiente limpo & Ajudar a manter limpo os espaços de prática \\
\hline 23 & $\begin{array}{l}\text { Ter uma boa relação com meu } \\
\text { professor/treinador }\end{array}$ & Ter uma boa relação com o meu professor/treinador \\
\hline 24 & Jogar de forma limpa & Trapacear/enganar \\
\hline 25 & Ter boas atitudes com meus colegas & Ter boas atitudes com os meus colegas \\
\hline 26 & Aceitar as decisões do(s) árbitro(s) & Aceitar as decisões dos árbitros \\
\hline 27 & $\begin{array}{l}\text { Cumprimentar os adversários no final do } \\
\text { jogo }\end{array}$ & Cumprimentar os adversários no final do jogo/prova \\
\hline 28 & Pedir desculpas quando necessário & Me desculpar quando faço algo errado \\
\hline 31 & Ter uma boa relação com os outros & Ter uma boa relação com os meus colegas \\
\hline
\end{tabular}


32 Entender-me com os outros

33 Buscar meu melhor nas aulas

37 Estar com meus colegas

38 Ajudar os colegas de escola

40 Apoiar meus colegas nos jogos

41 Buscar realizar corretamente habilidades

Me entender com os outros em uma situação de conflito/briga/discussão

Buscar meu melhor nas aulas/treinos

Interagir com os meus colegas

Ajudar os colegas de escola/equipe/grupo

Apoiar (torcer para) os meus colegas

as Buscar realizar corretamente os movimentos da modalidade

Nota. Elaborado pelos autores (2018).

Seguindo a avaliação do instrumento, no que diz respeito a abordagem qualitativa, as observações efetuadas pelos juízes permitiram um melhor refinamento em cada item. Com isto, foi possível realizar pequenos ajustes, em especial, naqueles itens em que foram pontuadas informações pertinentes à clareza ou à pertinência (Cassepp-Borges, Balbinotti \& Teodoro, 2010, Cassepp-Borges \& Teodoro, 2007).

Os cinco juízes-avaliadores apresentaram algumas sugestões nas observações e esses comentários foram analisados pelo grupo de pesquisadores para definir a modificação e lou ajuste do item. Dessa forma, a análise foi ampliada buscando a opinião dos juízes, a adequação da linguagem para um melhor entendimento das crianças (amostra da pesquisa) e os resultados do CVC, nos quesitos clareza e pertinência. A primeira versão dos itens, os resultados do CVC e as modificações estão apresentados no Quadro 2.

Alguns aspectos modificados merecem uma explanação e justificativa, conforme segue abaixo.

Muitas questões foram ajustadas tendo em vista a melhora na linguagem (inclusão de palavras, artigos, preposições) e na compreensão por parte do entrevistado, como o exemplo: 'Pedir desculpas quando necessário' do item 28, que foi alterado para 'Me desculpar quando faço algo errado'.

Outros itens precisaram de uma discussão maior, relativa a conceitos, conotação (se positivo ou negativo), foi optado por modificar o item 32 de 'Entender-me com os outros' para 'Me entender com os outros em uma situação de conflito/briga/discussão', procurando esclarecer o conceito abordado, como uma tentativa de não deixar dúvidas para a criança que responderá o questionário.

Durante a análise, por parte do grupo de pesquisadores, ficou evidenciado que existiam poucos itens negativos. Esse aspecto também foi mencionado por um juiz, na questão 24. Assim, definiu-se pela alteração dos itens, 13 e 25.

Um dos pontos de maior discussão foi no item 10. Esse item traz o conceito de habilidade, que pode não ficar claro para a criança que responderá o questionário. Pensando nisso, buscou-se estabelecer um critério e uma melhor definição deste conceito, o modificando para movimentos ligados à modalidade esportiva. Por este motivo, foi alterado também o item 15.

Alguns itens foram ajustados para que o ambiente ficasse definido, como a aula, o jogo, 0 treino, 0 campeonato, como aparece nas questões 5, 6, 27 e 38 como exemplo. Assim como no item 34, onde buscou-se separar o 'Fazer amigos' no ambiente de prática e aulas dos eventos esportivos.

\section{CONSIDERAÇÕES FINAIS}


Considera-se que os resultados do Coeficiente de Validade de Conteúdo (CVC) são adequados. $0 \mathrm{CVC}_{\mathrm{t}}$ (total) quanto à Pertinência e Clareza foram, respectivamente, 0,79 e 0,86. Os itens que apresentaram índices abaixo de 0,80 foram mantidos, pois entende-se que estes itens que compõem a versão original são importantes para a medida dos valores. Tais resultados permitem concluir que o IVO é um instrumento válido quanto à clareza e pertinência dos conteúdos dos itens.

Trata-se de um passo importante para a consolidação de um instrumento, confiável e que possa ser utilizado como um dos processos de avaliação nos diferentes projetos de Educação Olímpica no Brasil, enfatizando a possibilidade de ser uma ferramenta fundamental para o pleno desenvolvimento destes. Esse estudo não se encerra aqui. Novas análises devem ser realizadas, como por exemplo, validade de construto (fatorial exploratória e confirmatória) e de precisão (Alpha, Ômega). Para tanto, o presente estudo entrará em uma segunda etapa que será um estudo piloto para a aplicação do inventário a um grupo de 250 crianças de um projeto social esportivo certificado pelo Comitê Pierre de Coubertin.

\section{REFERÊNCIAS}

Abreu, N. (2002). Olympic multiculturalism: Proclaimed universal values versus cultural relativism. In DaCosta (Ed.), Olympic studies: Current intellectual crossroads (pp. 201-254). Rio de Janeiro, Brazil: Gama Filho.

Cassepp-Borges, V., Balbinotti, M. A. A., \& Teodoro, M. L. M. (2010). Tradução e validação de conteúdo: Uma proposta para a adaptação de instrumentos [Translation and content validation: A proposal for instruments adaptation]. In L. Pasquali (Ed.), Instrumentação psicológica: Fundamentos e práticas (pp. 506-520). Porto Alegre: Artmed.

Cassepp-Borges, V., \& Teodoro, M. L. (2007). Propriedades psicométricas da versão brasileira da Escala Triangular do Amor de Sternberg [Psychometrics Properties of the Brazilian Version of Sternberg's Triangular Love Scale], Psicologia: Reflexão e Crítica, 20(3), pp. 513-522. https://doi.org/10.1590/s0102-79722007000300020

Coluci, M. Z. O., Alexandre, N. M. C., \& Milani, D. (2015). Construção de instrumentos de medida na área da saúde [Construction of measurement instruments in the area of health], Ciência \& Saúde Coletiva, 20(3), pp. 925-936. https://doi.org/10.1590/1413-81232015203.04332013

Hernandez-Nieto, R. (2002). Contributions to statistical analysis. Mérida, Spain: Los Andes University Press.

Hill, M., \& Hill, A. (2008). Investigação por questionário [Questionnaire research]. Lisbon, Portugal: Sílabo.

International Olympic Committee [IOC]. (2007). The Olympic Symbols. Lausanne, Switzerland: Author.

Merlin, G. K., Lerina, D., Alves, A. R., Campos, T., Saldanha, R. P., Goularte, G. G., Fontoura, F. C., Faggiani, F., Ruskowski, J. S., Scarton, A. M., Todt, N. S. (2012). Educação Olímpica no Brasil: Construção, Validação e Consistência Interna do "Inventário de Valores Olímpicos" [Olympic Education in Brazil: Construction, Validation and Internal Consistency of the "Inventory of Olympic Values"]. In XIII Salão de Iniciação Científica PUCRS. Porto Alegre. XIII Salão de Iniciação Científica PUCRS. Porto Alegre, Brazil: EDIPUCRS. https://doi.org/10.5783/rirp-9-2015-05-67-88

Merlin, G. K., Todt, N. S., Scarton, A. M., Saldanha, R. P. (2016a). Content validity of the 'Olympic Values Inventory'. In Mataruna et al. (Eds.), $2^{\circ}$ Simpósio Internacional Pierre de Coubertin (pp. 51-52). Rio de Janeiro, Brazil: EDIPUCRS. Retrieved from http://coubertin.org/docs/2SymposiumBras2016.pdf

Merlin, G. K., Todt, N. S., Scarton, A. M., Saldanha, R. P. (2016b). Content validity of the 'Olympic Values Inventory'. In Proceedings of the Thirteenth International Symposium for Olympic Research. Vitória, 
Brazil. The future of the Olympic Event in the Age the Mega-Spectacle (pp. 47-48). London, Ontario, Canada, N6A5B: International Centre for Olympic Studies- Western University Canada. https://doi.org/10.14198/ihse.2018.13.proc1.13

Secco, V. G., Santos, F., Costa, C. P., Saldanha, R. P., Rolim Silva, L. H., Todt, N. S. (2009). Educação Olímpica: avaliação da validade de conteúdo do "Questionário de Valores Olímpicos" (QVO-27) [Olympic Education: evaluation of the contente validation of the 'Olympic Values Questionnaire']. In X Salão de Iniciação Científica PUCRS. Porto Alegre. X Salão de Iniciação Científica PUCRS. Porto Alegre, Brazil: EDIPUCRS. https://doi.org/10.15689/ap.2017.1602.03

Moraes, C. L., Hasselmann, M. H., \& Reichenheim, M. E. (2002). Adaptação transcultural para 0 português do instrumento "Revised Conflict Tactics Scales (CTS2)" utilizado para identificar violência entre casais [Portuguese-language cross-cultural adaptation of the Revised Conflict Tactics Scales (CTS2), an instrument used to identify violence in couples], Cadernos de Saúde Pública, 18(1), pp. 163-176. https://doi.org/10.1590/s0102-311x2002000100017

Müller, N., \& Todt, N. (2015). Pierre de Coubertin (1863-1937), Olimpismo: seleção de textos [Pierre de Coubertin (1863-1937), Olympism: selected writtings]. Porto Alegre, Brazil: EDIPUCRS. https://doi.org/10.1093/ww/9780199540884.013.u207930

Pasquali, L. (2001). Técnicas de exame psicológico-TEP: Manual [Psychological exam techniques: Manual]. São Paulo, Brazil: Casa do Psicólogo.

Perron, J. (1987). Base e aplicação dos valores em psicologia e educação [Bases and application of the values in psychology and education]. Porto Alegre, Brazil: Sagra Editora.

Rokeach, M. (1981). Crenças, atitudes e valores [Believes, attitudes and values]. Rio de Janeiro, Brazil: Interciência.

Saldanha, R. P., Balbinotti, M. A. A., \& Balbinotti, C. A. A. (2015). Tradução e validade de conteúdo do Youth Sport Value Questionnaire 2 [Translation and validity content of Youth Sport Value Questionnaire 2], Revista Brasileira de Ciências do Esporte, 37(4), pp. 383-388. https://doi.org/10.1016/j.rbce.2015.08.010

Scarton, A. M., Todt, N. S., Merlin, G. K., Saldanha, R. P. (2016). Content validity of the 'Olympic Values Inventory'. In Second International Colloquium of Olympic Studies and Research Centres: Booklet of Abstracts (pp. 20-20). Porto Alegre, Brazil: EDIPUCRS. https://doi.org/10.18411/d-2016-154

Schwartz, S. H. (2007). Universalism values and the inclusiveness of our moral universe, Journal of Cross-Cultural Psychology, 38(6), pp. 711-728. https://doi.org/10.1177/0022022107308992

Schwartz, S. H., \& Cieciuch, J. (2016). Values. In Miller, H. L. (Ed.), The SAGE Encyclopedia of Theory in Psychology (pp. 949-951). Thousand Oaks, United States: SAGE.

Todt, N. S. (2015). The Olympic Education programs acknowledged by the Brazilian Pierre de Coubertin Committee: A stakeholder model taking shape in Brazil. In Deslandes, A., DaCosta, L., \& Miragaya, A. (Eds.), The Future of Sports Mega-Events (pp. 415-420). Rio de Janeiro, Brazil: Engenho Arte e Cultura.

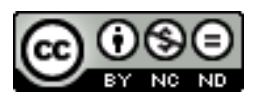

This work is licensed under a Attribution-NonCommercial-NoDerivatives 4.0 International (CC BY-NC-ND 4.0). 